\title{
Formularmäßige Verkürzung der Veriährungsfrist für Schadensersatzansprüche gegen den Tierarzł wegen fehlerhafter Kaufuntersuchung
}

\author{
Dietrich Plewa \\ Kanzlei Dr. Plewa und Doppler, Germersheim
}

In den bis zum 31.12.2001 gebräuchlichen Formularen zur Kaufuntersuchung, herausgegeben vom Hippiatrika-Verlag, war formularmäßig die Verjährungsfrist für Schadensersatzansprüche gegen den Tierarzt auf sechs Monate beschränkt. Die durch die allgemeinen Geschäftsbedingungen des Tierarztes (AGB) vorgesehene Verjährungsregelung wich damit erheblich von derjenigen $a b$, die nach einer Grundsatzentscheidung des Bundesgerichtshofes aus dem Jahr 1983 auf Schadensersatzansprüche wegen fehlerhafter Kaufuntersuchung anzuwenden war, nämlich die bis Ende 2001 geltende allgemeine Veriährungsfrist von 30 Jahren (BGH 1983). Durch mehrere Urteile war die Wirksamkeit der formularmäßigen Verkürzung der Veriährungsfrist bestätigt worden (OLG Oldenburg 1998; OLG München 1996). Diese Rechtsauffassung ist zuletzt noch durch das Oberlandesgericht Koblenz mit einem Urteil vom 25.02.2003 (AZ: 3 U 1076/02) bestätigł worden. Aus der letz† genannten Entscheidung könnten falsche Schlüsse gezogen werden. Da sie im Jahr 2003 erlassen wurde, könnte der juristische Laie auf die Idee kommen, die Entscheidung habe auch Bedeutung für das neve Schuldrecht, das zum 01.01.2002 in Kraft getreten ist. Das jedoch wäre ein möglicherweise folgenschwerer Irrtum: Das Urteil des OLG Koblenz befasst sich mit einer Kaufuntersuchung, die im Jahr 2001 durchgeführt worden war. Die rechtliche Beurteilung hatte sich daher am alten Schuldrecht zu orientieren. Nach den Überleitungsvorschriften kommt es für die Frage der Anwendbarkeit des Rechts auf den Zeitpunkt des Vertragsabschlusses an. Dies gilt übrigens für das neue Kaufrecht nicht anders wie für die Bestimmungen zum Werkvertrag, die für die Beurteilung der Kaufuntersuchung einschlägig sind. Die Entscheidung des OLG Koblenz gibł daher für die aktuelle Gesetzeslage nichts her.

Die regelmäßige Veriährungsfrist wurde von 30 Jahren auf drei Jahre verkürzt, § 195 BGB. Der Vertrag über die Kaufuntersuchung ist nach einheitlicher Auffassung als Werkvertrag zu qualifizieren (BGH 1983). Daher ist für die Verjährung $\S 634 a$ BGB neve Fassung einschlägig. Diese Bestimmung gilt für alle Mängelrechte, auch für Schadensersatzansprüche im Verhältnis zwischen Tierarzł und Auftraggeber. Zu bedenken ist allerdings, dass es für die Schadensersatzpflicht des Tierarztes unerheblich ist, ob der Auftrag zur Kaufuntersuchung durch den Käufer oder Verkäufer erteilt wurde. Zwar bestehen vertragliche Schadensersatzansprüche regelmäßig nur zwischen den Vertragsparteien, etwas anderes gilt aber dann, wenn ein Dritter in den Schutzbereich des Vertrages einzubeziehen ist. Das ist von der Rechtsprechung regelmäßig für die Position des Käufers bejaht worden, wenn Auftraggeber des Tierarztes für die Kaufuntersuchung der Verkäufer war, dem Tierarzł aber bekannt gewesen war, dass die Kaufentscheidung von dem Ergebnis der Untersuchung abhängt (OLG Köln 1992; OLG Düsseldorf 1998).

Gemäß § 634a Abs.1 Z.3 BGB verjähren alle Ansprüche gegenüber dem Tierarzt in der regelmäßigen Verjährungsfrist, also innerhalb von drei Jahren. Diese Frist gilt, wenn nicht ausdrücklich etwas anderes vereinbart ist. Der Gestaltungsfreiheit sind allerdings Grenzen gesetzt. Während nach der früheren Gesetzeslage die Verkürzung auf sechs Monate zulässig war, darf seit der Schuldrechtsreform in einem Formularvertrag die Veriährung nicht auf weniger als ein Jahr verkürzt werden, $\S 309$ Nr.8b BGB. Zu beachten ist dabei, dass das Verbot einer Abkürzung der Veriährung auf weniger als ein Jahr auch mittelbare Verschlechterungen beinhaltet wie etwa die Vorverlegung des Veriährungsbeginns (BGH 1987; Palandt/Heinrichs). Dies bedeutet, dass es bei dem gesetzlichen Veriährungsbeginn zu verbleiben hat. Diesen definiert $\S 199$ dahingehend, dass die regelmäßige Veriährungsfrist mit dem Schluss des Jahres beginnt, in dem

- der Anspruch entstanden ist und

- der Gläubiger von den den Anspruch begründenden Umständen und der Person des Schuldners Kenntnis erlangt oder ohne grobe Fahrlässigkeit erlangen müsste.

Diese Bestimmung zum Veriährungsbeginn ist zwingend und kann nicht durch AGB aufgehoben werden. Sie bedeutet, dass die Veriährung erst beginnt, wenn der Käufer Anhaltspunkte dafür hat oder haben muss, dass der Tierarzt die Kaufuntersuchung fehlerhaft durchgeführt, also beispielsweise einen vorhandenen Befund übersehen oder einen gebotenen Untersuchungsschritt nicht durchgeführt hat. Insoweit ist selbst die einjährige Veriährungsfrist, die in $A G B$ vorgesehen werden kann, letztlich nicht relevant, da sie abhängig ist von der Kenntnis des Käufers davon, dass möglicherweise Schadensersatzansprüche gegeben sind. Unabhängig von dieser Kenntnis veriähren die Schadensersatzansprüche erst in zehn Jahren von der Entstehung der Forderungen an, § 199 Abs.3 
Z.1 BGB. Diese - kenntnisunabhängige - Veriährungsfrist wiederum kann formularmäßig nur auf eine Frist von fünf Jahren verkürz† werden (Plewa 2002).

Die Rechtsauffassung, der Tierarzt könnte in einem Formularvertrag die Veriährungsfrist für Schadensersatzansprüche aus einer fehlerhaften Kaufuntersuchung auf sechs Monate beschränken, ist nach alledem schlicht falsch. Der geltenden Gesetzeslage trägt das aktuelle Formular des Hippiatrika-Verlages Rechnung.

\section{Literatur}

BGH (1983): NJW 83, 2078

BGH (1987): NJW-RR 87, 145;
OLG Düsseldorf (1998): AZ: 8 U 151/98

OLG Köln (1992): VersR 92, 978

OLG München (1996): VersR 96, 731

OLG Oldenburg (1998): VersR 98, 1563;

Palandt/Heinrichs: BGB, Bürgerliches Gesetzbuch, 64. Auflage, München 2005, Rz 75 zu $\S 309$

Plewa D. (2002): Pferdeheilkunde 18, 284-288

Dr. jur. Dietrich Plewa

Ludwig-Erhard-Straße 4

76726 Germersheim

rae.plewa-doppler@t-online.d

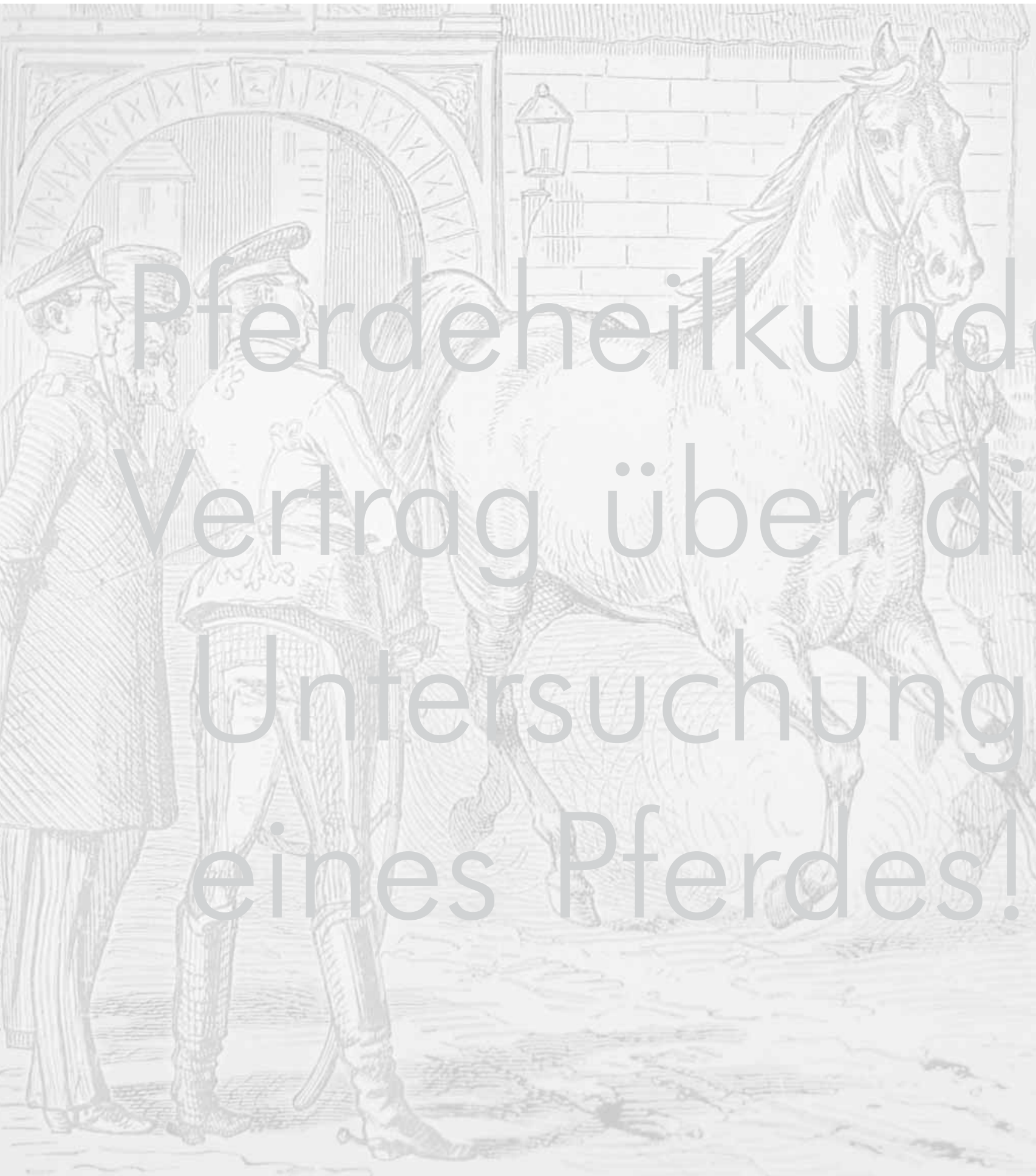

